Proceeding Series of the Brazilian Society of Computational and Applied Mathematics

\title{
Localização de Atuadores Piezelétricos com Efeito Cisalhantes
}

Odair Menuzzi ${ }^{1}$

Departamento Acadêmico de Matemática, IFFARROUPILHA, São Borja, RS

Eduardo Padoin

Departamento Acadêmico de Matemática, IFFARROUPILHA, Alegrete, RS

Jun Sérgio Ono Fonseca 3

Departamento de Engenharia Mecânica, UFRGS, Porto Alegre, RS

Eduardo André Perondi ${ }^{4}$

Departamento de Engenharia Mecânica, UFRGS, Porto Alegre, RS

Otavio Augusto Alves da Silveira

Departamento de Engenharia Civil, UFSC, Florianópolis, SC

\begin{abstract}
Resumo. Este trabalho busca desenvolver uma metodologia de projeto ótimo para localização de material piezelétrico com efeito cisalhante para atenuar vibrações em estruturas tridimensionais. $\mathrm{O}$ objetivo é determinar a localização ótima de atuadores através do processo de otimização topológica. A localização dos atuadores é determinada por uma otimização que define onde o material deve ter propriedades piezelétricas; através da maximização da controlabilidade, medida por seu gramiano. Resultados preliminares mostram a eficácia do processo de otimização topológica quanto à localização dos atuadores na estrutura.
\end{abstract}

Palavras-chave. Otimização topológica; material piezelétrico; efeito cisalhante.

\section{Introdução}

Há algum tempo, cientistas vêm pesquisando materiais que equipados com atuadores, sensores e sistemas de controle de alto desempenho, se comportam como sistemas inteligentes. Esses tipos de materiais inteligentes já têm grande sucesso no meio acadêmico. Em breve, materiais que se consertam adaptam a certas condições do meio ambiente poderão estar mais acessíveis. Além disso, existem altos investimentos por parte de grandes

1 odair.menuzzi@iffarroupilha.edu.br

2 eduardo.padoin@iffarroupilha.edu.br

3 jun@ufrgs.br

4 eduardo.perondi@ufrgs.br

5 otavio.silveira@ufsc.br 
indústrias e do Estado para esses estudos.

As principais aplicações dos materiais inteligentes se dão na indústria espacial e aeroespacial, mas não menos importante em áreas como veicular, biomédica e robótica [9], onde o objetivo é o alto desempenho, através de estruturas que têm grande capacidade de automonitoramento e controle ativo. Dessa forma, é importante a utilização das mais avançadas técnicas para o estudo e melhoramento dessas estruturas inteligentes.

$\mathrm{O}$ uso desses materiais inteligentes obteve muitos avanços nas últimas décadas e ainda percebe-se grande potencial e possibilidade de inovações, principalmente quando se utilizam, métodos como a otimização topológica, que contribui para o projeto de estruturas mais leves, diminuindo os custos. Contudo, reduzir o peso e alterar o amortecimento de uma estrutura pode gerar alguns problemas, como, por exemplo, o aparecimento de vibrações excessivas. Diante disso, é importante a utilização de um controle ativo ligados por um sistema de realimentação. Um sistema realimentado tem a capacidade de reduzir a sensibilidade da saída em relação a mudanças nos parâmetros.

Recentemente, [11] abordou o problema de otimização topológica de pares de sensores e atuadores piezelétricos para o controle de vibração torcional de uma placa laminada, [2] utilizou a otimização topológica num projeto simultâneo para estrutura e localização de atuadores em estruturas flexíveis, [4] estudaram a otimização topológica de atuadores de flexão com material piezelétrico multicamada.

A localização adequada de atuadores piezelétricos, de acordo com [5] influencia de maneira positiva no desempenho do sistema de controle e da resposta controlada. $\mathrm{Na}$ área de controle ótimo foi encontrado um número grande de publicações. [3] abordaram o uso de materiais inteligentes baseados numa teoria de deformação de cisalhamento de primeira ordem. Foram estudadas características de uma viga descontínua com sensores e atuadores distribuídos. Foi aplicado também um regulador LQR para reduzir as vibrações na viga usando o método de integração direta de alta precisão. [12] apresentaram um estudo sobre a otimização topológica de atuadores piezelétricos planares montados com padrões repetitivos, onde o objetivo é maximizar a concepção do trabalho efetuado pelo deslocamento da saída, enquanto que as restrições são impostas sobre o gasto da energia de atuação e sobre o volume de material. [10] desenvolveram um projeto simultâneo para a topologia estrutural e localização de atuadores, a qual é determinada topologicamente como um subprocesso, através da maximização de uma medida de controlabilidade. Além disso, é abordado o controle LQR para redução de vibrações. Outras importantes publicações que trabalham com nesta área são $[2,5]$.

\section{Modelo de Elementos Finitos em Espaço de Estados}

O modelo utilizado para o problema é dado pela equação de elementos finitos global que governa o movimento espacial e o equilíbrio de cargas elétricas dado pela Equação 1, escrita como:

$$
\begin{gathered}
\boldsymbol{M}_{u u} \ddot{\boldsymbol{u}}+\boldsymbol{H}_{u u} \boldsymbol{u}+\boldsymbol{H}_{u p} \boldsymbol{\phi}_{e}=\boldsymbol{f}, \\
\boldsymbol{H}_{u p}^{T} \boldsymbol{u}+\boldsymbol{H}_{p p} \boldsymbol{\phi}_{e}=\boldsymbol{q}_{e},
\end{gathered}
$$

onde os vetores globais dos graus de liberdade mecânico e elétrico são $\boldsymbol{u}$ e $\boldsymbol{\phi}_{e}$, respectivamente; a matriz global de massa é dada por $\boldsymbol{M}_{u u}$; a matriz de rigidez condensada é dada por $\boldsymbol{H}_{u u}$; as matrizes globais de acoplamento piezelétrico são $\boldsymbol{H}_{u p}=\boldsymbol{H}_{u p}^{T} ; \boldsymbol{H}_{p p}$ é a matriz condensada dielétrica; $\boldsymbol{f}$ é o vetor global externo de forças mecânicas e $\boldsymbol{q}_{e}$ é o vetor global condensado de cargas elétricas.

Os graus de liberdade elétricos serão utilizados como atuantes e conhecidos, ou seja, 
como entradas para o atuador no sistema de controle. Isso se deve ao fato de que os graus de liberdade no eletrodo aterrado foram desconsiderados por não apresentar potencial elétrico, além dos graus de liberdade internos terem sido condensados [1].

A segunda equação da Equação 1 pode ser utilizada para determinar a carga elétrica nos eletrodos, uma vez que, os eletrodos são aterrados, de forma que $\phi_{e}=0$.

Como já citado, um dos problemas da utilização do modelo modal truncado, onde são considerados apenas alguns modos de vibração, é que pode surgir o problema de spillover, ou seja, a excitação dos modos residuais pelo sistema de controle. Diante

disso, pode-se considerar que uma matriz modal truncada pode ser utilizada na transformação de coordenadas generalizadas $\boldsymbol{u}$ para modais $\boldsymbol{\eta}$, onde o vetor de deslocamentos pode ser aproximado pela superposição dos $m$ primeiros modos, conforme a Equação 2.

$$
\boldsymbol{u}=\boldsymbol{\Psi} \boldsymbol{\eta} \cong \sum_{i=1}^{m} \Psi_{i} \eta_{i},
$$

onde, $\boldsymbol{\Psi}=\left[\Psi_{1}, \ldots, \Psi_{m}\right]$ é a matriz modal truncada e $\boldsymbol{\eta}=\left\{\eta_{1}, \ldots \eta_{m}\right\}^{T}$ é o vetor de coordenadas modais correspondente. Com essa transformação, a ordem do sistema passa a ser o número de modos que representa o modelo modal e não mais o número de graus de liberdade do modelo de elementos finitos.

Para utilizar-se uma notação mais usual, a Equação 1 é transformada na Equação 3, que representa o modelo modal truncado de elementos finitos com atuadores piezelétricos, onde é considerado um modelo de amortecimento viscoso simples:

$$
\begin{gathered}
\ddot{\boldsymbol{n}}+2 \boldsymbol{Z} \boldsymbol{\Omega} \dot{\boldsymbol{n}}+\boldsymbol{\Omega}^{2} \boldsymbol{n}=-\boldsymbol{\Psi}^{\mathrm{T}} \boldsymbol{H}_{u p} \boldsymbol{\phi}_{e}+\boldsymbol{\Psi}^{\mathrm{T}} \boldsymbol{f}, \\
\boldsymbol{y}=\boldsymbol{H}_{u p}^{T} \boldsymbol{\Psi} \boldsymbol{n},
\end{gathered}
$$

onde $\boldsymbol{\Omega}$ é a matriz diagonal de frequências naturais e $\boldsymbol{Z}$ é a matriz diagonal de amortecimento modal para os $m$ modos considerados.

Para obter o modelo em espaço de estados, inicialmente se define o vetor de estados em função dos deslocamentos ou velocidade modais do modelo truncado. Com isso, chegase ao sistema em malha fechada, dado por uma equação diferencial matricial de primeira ordem expressa em termos do vetor de variáveis de estado.

$$
\begin{gathered}
\dot{\boldsymbol{x}}=\boldsymbol{A} \boldsymbol{x}+\boldsymbol{B}_{\phi} \boldsymbol{u}_{\phi}^{c}+\boldsymbol{B}_{u} \boldsymbol{u}_{u}^{c}, \\
\boldsymbol{y}=\boldsymbol{C}^{c} \boldsymbol{x},
\end{gathered}
$$

onde a matriz do sistema é dada por $\boldsymbol{A}$, as matrizes de entradas mecânicas e elétricas são dadas por $\boldsymbol{B}_{u}$ e $\boldsymbol{B}_{\phi}$, respectivamente, $\boldsymbol{C}^{c}$ é a matriz de saída e os vetores de entradas mecânicas e elétricas são dados por $\boldsymbol{u}_{u}^{c}$ e $\boldsymbol{u}_{\phi}^{c}$, respectivamente, conforme:

$$
\boldsymbol{A}=\left[\begin{array}{cc}
\mathbf{0} & \boldsymbol{I} \\
-\boldsymbol{\Omega}^{2} & -2 \boldsymbol{Z} \boldsymbol{\Omega}
\end{array}\right], \quad \boldsymbol{B}_{\phi}=\left[\begin{array}{c}
\mathbf{0} \\
-\boldsymbol{\Psi}^{\mathrm{T}} \boldsymbol{H}_{u p}
\end{array}\right], \quad \boldsymbol{B}_{u}=\left[\begin{array}{c}
\mathbf{0} \\
\boldsymbol{\Psi}^{\mathrm{T}}
\end{array}\right] \text { e } \boldsymbol{C}^{c}=\left[\begin{array}{lll}
\boldsymbol{H}_{u p}^{T} \boldsymbol{\Psi} & \mathbf{0}
\end{array}\right] .
$$

\subsection{Gramiano de controlabilidade}

O conceito de controlabilidade apresenta duas dificuldades. Em primeiro lugar, a resposta sempre é dada em termos de "sim" ou "não", ou seja, uma resposta qualitativa e não quantitativa sobre o problema. Segundo, apresenta problemas numéricos quando submetidos a sistemas com grandes dimensões. Diante disso, uma solução alternativa para transpor esta dificuldade é a formulação em termos de Gramiano, que não sofrem os problemas citados, já que são dados de forma quantitativa. O Gramiano caracteriza se o sistema é controlável (observável) ou não. Conforme [7], se um sistema é assintoticamente estável, ou seja, se todos os polos da matriz A tem parte real negativa, a resposta do sistema é limitada e a 
matriz de covariância para estado estável é finita. Então, a resposta para um grupo de ruídos brancos independentes é:

$$
\boldsymbol{W}_{c}=E\left[\boldsymbol{x} \boldsymbol{x}^{T}\right]=\int_{0}^{\infty} e^{A \tau} \boldsymbol{B} \boldsymbol{B}^{T} e^{\boldsymbol{A}^{T} \tau} d \tau,
$$

onde $\boldsymbol{W}_{c}$ é conhecido como Gramiano de controlabilidade e $E$ é o operador de covariância. Pode-se ainda obter o Gramiano de controlabilidade através da equação de Lyapunov.

$$
\boldsymbol{A} \boldsymbol{W}_{c}+\boldsymbol{W}_{c} \boldsymbol{A}^{T}+\boldsymbol{B} \boldsymbol{B}^{T}=\mathbf{0}
$$

O sistema é controlável se todos os estados do sistema podem ser excitados pela entrada de controle. Esta condição é satisfeita se $\boldsymbol{W}_{c}$ é positiva definida [7].

\section{Otimização Topológica}

A localização ótima dos atuadores pode auxiliar para a redução de vibrações em estruturas. $\mathrm{O}$ sistema de controle é projetado com uma função objetivo que maximiza o traço do Gramiano de Controlabilidade, onde o índice ótimo é:

$$
f_{a}\left(\rho_{a}, \rho_{s}\right)=f_{a}=\operatorname{tr}\left(\boldsymbol{W}_{c}\right)
$$

$\boldsymbol{W}_{c}$ é o Gramiano de Controlabilidade. Diante disso, a otimização de controle é escrita da seguinte forma:

$$
\text { sujeito a }\left\{\begin{array}{c}
\max _{\rho_{a}} f_{a}\left(\rho_{a}, \rho_{s}\right), \\
0<\rho_{a i} \leq 1, \quad\left(i=1,2, \ldots, N_{a}\right), \\
V_{a}=\frac{\int_{\Omega} \rho_{a i} d \Omega}{\int_{\Omega} d \Omega} \leq V_{a}^{\max },
\end{array}\right.
$$

onde $\rho_{a i}$ e a i-ésima componente do vetor de variáveis de projeto de controle para localização de atuadores, $N_{a}$ é o número de variáveis de projeto de controle para localização dos atuadores que é igual ao número de elementos finitos, e a segunda restrição limita o volume total de material piezelétrico para o atuador a uma fração do volume $V_{a}^{\max }$ préestabelecida. A resolução dos problemas de otimização podem ser resolvidos por qualquer método de programação matemática. Contudo, neste trabalho utilizou-se o método da programação linear sequencial, a fim de testar a metodologia proposta. Além disso, o modelo material e a sensibilidade do gramiano de controlabilidade são semelhantes ao trabalho de [10].

\section{Resultados}

Na Figura 1 é apresentado uma estrutura tipo viga em balanço com medidas $440 \mathrm{~mm}$ x $110 \mathrm{~mm}$ x $10 \mathrm{~mm}$, discretizada em 484 elementos cúbicos. Nesse caso, considera-se restrições devidas ao engaste. Os resultados obtidos utilizando as estratégias propostas foram implementados em MATLAB. Em todas as simulações as estruturas são discretizadas em elementos finitos sólidos isoparamétricos de 8 nós, com três graus de liberdade mecânicos e um grau de liberdade elétrico por nó. A proposta do trabalho busca a otimização do sistema de controle pela maximização do traço do gramiano de controlabilidade, usado para a localização de atuadores na estrutura. As propriedades mecânicas do material elástico isotrópico (Alumínio) e do material piezelétrico (PZT5A) consideradas nas análises foram obtidos a partir dos trabalhos de [6] e [8]. 


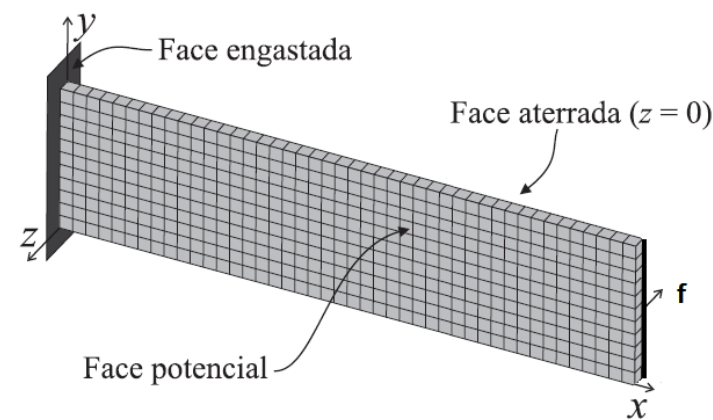

Figura 1 - Viga em balanço discretizada em 484 elementos finitos sólidos isoparamétricos $(44 \times 11 \times 1)$.

Para o projeto de otimização, a restrição de volume piezelétrico é $5 \%$. As pseudodensidades são uniformes para todos os elementos, sendo iguais a $\rho_{a}=0.05$. Desta forma, o problema de otimização se inicia logo abaixo do limite viável. O critério de parada estabelece a modificação das variáveis de projeto de uma iteração para outra for menor que $2 \%$. Os coeficientes de penalização do modelo material foram $3 \mathrm{em}$ todos os casos analisados. Nenhuma técnica de continuação foi utilizada.

A Figura 2 mostra o comportamento para o efeito cisalhante.

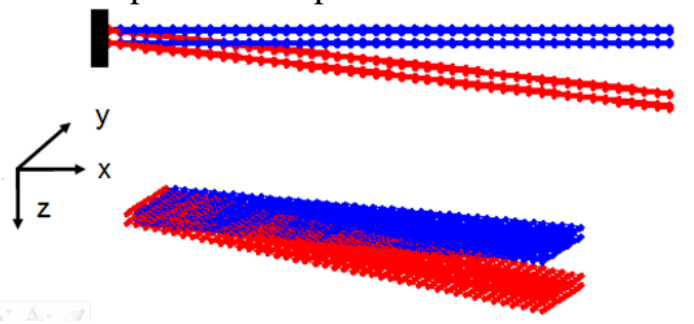

Figura 2 - Efeito cisalhante.

A topologia ótima para a distribuição de material piezelétrico analisando o primeiro modo de vibração é apresentada na Figura 3. A cor em preto representa a localização dos atuadores na estrutura.

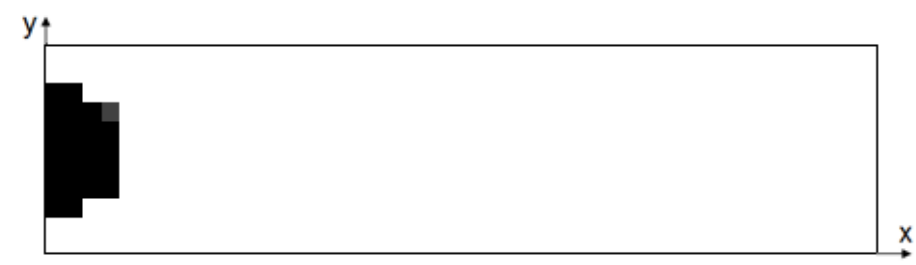

Figura 3 - Topologia para distribuição de material piezelétrico para o $1^{\circ}$ modo de vibração.

Na Figura 3, pode-se observar que os resultados para localização dos atuadores se dá na parte próxima ao engaste da viga, onde conforme o modo de vibração tem-se a maior influencia do respectivo modo.

A convergência da função objetivo é importante para verificar o comportamento da otimização com o passar das iterações, salientando que os valores iniciais das variáveis de projeto são iguais para todas as topologias. Na Figura 4 podem ser vistos o histórico da convergência da função objetivo para otimizaçao dos atuadores. 


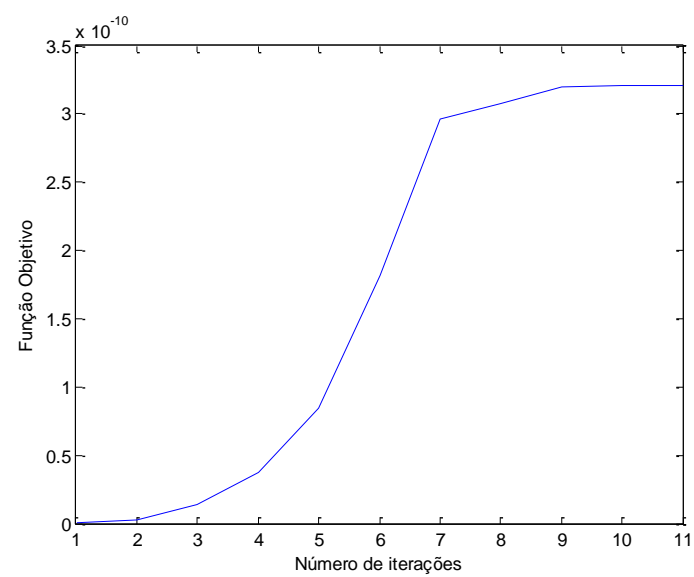

Figura 4 - Convergência da função objetivo analisando o $1^{\circ}$ modo de vibração

\section{Conclusão}

Resultados de testes usando a metodologia proposta, levando em consideração o efeito cisalhante, mostraram que a localização ótima dos atuadores está significativamente consistente com o modo de vibração analisado. Ademais, os resultados da convergência das funções objetivo para os atuadores ao longo do processo de otimização mostraram um comportamento regular do algoritmo de otimização.

\section{Agradecimentos}

Os autores agradecem ao apoio financeiro da Capes e CNPq e também a UFRGS.

\section{Referências}

[1] J. Becker, O. Fein, M. Maess e L. Gaul, Finite element-based analysis of shunted piezoelectric structures for vibration damping, Computers and Structures, vol. 84, p. 2340-2350, 2006.

[2] R.C. Carbonari, E. C. N. Silva e S. Nishiwaki, Optimum placement of piezoelectric material in piezoactuator design, S.M.S., 2007.

[3] F. Chen, M. Hong, M. Song e H. Cui, Optimal Control of a Beam with Discontinuously Distributed Piezoelectric Sensors and Actuators, Journal of Marine Science and Application, (2012).

[4] Z. Kang e X. Wang, Topology optimization of bending actuators with multilayer piezoelectric material, Smart Material and Structures, (2010).

[5] R. Kumar e S. Narayanan, Active vibration control of beams with optimal placement of piezoelectric sensor/actuator pairs, Smart Materials and Structures, vol. 17, p. 01-15, (2008).

[6] A. Mecchi, G. Nader, E. Silva e J. Adamowski, Development and Characterization of a 
Unimorph-type Piezoelectric Actuator Applied to a Michelson Interferometer, ABCM Symposium Series in Mechatronics, vol. 01, (2004).

[7] A. Preumont, Vibration Control of Active Structures, An Introduction. Kluwer, (2002).

[8] W. Rubio, E. Silva e G. Paulino, Toward optimal design of piezoelectric transducers based on multifunctional and smoothly graded hybrid material systems, Journal of Intelligent Material Systems and Structures, vol. published online 31 July, (2009).

[9] M. Schwantz, Encyclopedia of smart materials. Vol. 1 e Vol. 2. A Wiley-Interscience Publication, (2002).

[10] O. A. A da Silveira, J. S. O. Fonseca e I. F. Santos, Actuator topology design using the controllability Gramian, Struct Multidisc Optim, (2014).

[11] S. Y. Wang, K. Tai e S. T. Quek, Topology optimization of piezoelectric sensors/actuators for torsional vibration control of composite plates, Smart Material and Structures, (2006).

[12] X. Wang, Z. Kang e Y. Wang, Topology design of slender piezoelectric actuators with repetitive component patterns. Journal of Intelligent Material Systems and Structures, (2011). 